\title{
SIMO Diversity with Antenna Selection for DVB-S2/RCS in LMS Scenarios
}

\author{
S. Cioni, A. Vanelli-Coralli and G.E. Corazza \\ DEIS-ARCES, University of Bologna \\ Via V. Toffano, 2/2, Bologna, Italy \\ scioni@deis.unibo.it, avanelli@arces.unibo.it, \\ gecorazza@deis.unibo.it
}

\author{
J.L. Vicario and M. A.Vázquez Castro \\ Dpt. Telecom and Systems Engineering \\ 2Universitat Autònoma de Barcelona \\ Bellaterra, Spain \\ jose.vicario@uab.es, angeles.vazquez@uab.es
}

\begin{abstract}
In this paper, we propose a Single-Input MultipleOutput (SIMO) diversity technique to improve the link reliability of a satellite communications system. In particular, the DVB-S2 communication system is investigated in a land mobile satellite environment. Realistic physical layer simulations are carried out to better evaluate the possible gain of using multiple receiving antennas at the receiver side. A hybrid selection technique of the available antennas is presented to reduce the complexity and the cost of the user terminal.
\end{abstract}

Keywords - antenna diversity; antenna selection; DVB-S2; LMS scenarios

\section{INTRODUCTION}

New satellite communications systems are expected to provide broadband services to mobile users terminals. In order to take advantage of the current technology, it is of great interest to study the viability of adopting the DVB-S2 standard [1] in a land mobile satellite (LMS) scenario. The challenge introduced by the LMS environment appears due to the mobility, in terms of the Doppler effect, the possible loss of the line-of-sight and the time-variant nature of the channel.

Recently, several authors have proposed spatial diversity techniques at the receive side, i.e., Single Input Multiple Output (SIMO), in order to improve system reliability in LMS scenarios [2], [3]. In those papers, the authors considered a railway scenario, where broadband services were provided to high-speed trains by means of a satellite communications system. In that case, the negative effects of the mobile channel plus the presence of periodic deep fading events due to the presence of power arches could be efficiently compensated with the help of the second receiving antenna. However, when multi-antenna techniques are adopted in an urban or sub-urban scenario with mobile user terminals (i.e., handhelds or laptops), it should be considered that these devices are limited in size, complexity and cost. The problem of exploiting SIMO diversity and of keeping a low level of complexity in these kinds of devices was addressed in [4]. Among the several discussed techniques, one proposed solution was the adoption of a Hybrid Selection-Maximal-Ratio Combining (HS-MRC) strategy [4]. In a HS-MRC system, the system is equipped with $N$ receive antennas but only $L<N$ complete $\mathrm{RF}$ chains are available. The main idea consists in selecting the best sub-set of $L$ out of $N$ receive antennas for reception. After that, the received signals are combined by means of a $\mathrm{MRC}$ procedure.
This scheme was shown to be very effective in wireless networks as most of the diversity gain obtained with the full antenna system can be extracted but only $L$ complete RF chains are needed and, thus, receiver complexity, size and hardware cost are not substantially increased.

In this paper, we adopt the HS-MRC strategy to improve system efficiency in LMS scenarios. More specifically, we analyze performance of HS-MRC in a satellite communication system based on DVB-S2. To do so, we take into consideration a realistic situation where the complete forward-link transmit receive chain is adopted. Furthermore, we also analyze the effects of realistic algorithms for symbol timing acquisition, channel state estimation, carrier frequency and phase offset recovery.

The remainder of the paper is organized as follows. The corresponding signal and system model is presented in Section II. Next, we introduce the Hybrid Selection/MRC strategy in detail in Section III. After that, we present the digital receiver architecture in Section IV, devoting particular attention to the SNR estimation block which is in charge to select the best $L$ receive antennas. Finally, Section $\mathrm{V}$ shows the numerical results obtained in Rayleigh fading for several combinations of $N$ and $L$, whereas Section VI draws the conclusion and future development.

\section{SYSTEM MODEL}

Consider the forward link of a satellite communications system based on DVB-S2 [1], where broadband services are provided to mobile user terminals in a sub-urban scenario. In such a scenario, the channel response can be modeled by combination of LOS and NLOS statistical models, being each of them Ricean distributed according to the user speed. In order to improve link reliability, a multiple-antenna system is adopted at the receiver. Further details of the proposed system are presented below.

\section{A. Transmitter}

The considered DVB-S2 physical layer transmitter [1] is depicted in Figure 1. A continuous stream of MPEG packets form the baseband frame (BBFRAME) ready to be encoded. Each BBFRAME is processed by the Forward Error Correction (FEC) encoder which is carried out by the concatenation of a Bose-Chaudhuri-Hocquenghem (BCH) outer code and a LDPC inner code. Available code-rates for the inner code are $1 / 4,1 / 3$, 
$2 / 5,1 / 2,3 / 5,2 / 3,3 / 4,4 / 5,5 / 6,8 / 9$, and $9 / 10$. Depending on the application area, codewords can have length $N_{L D P C}=64800$ bits or 16200 bits. In the following, the case of 64800 bits is considered. The possible modulation formats proposed in the DVB-S2 standard are QPSK, 8PSK, 16APSK or 32APSK constellations. The modulated stream enters in the physical layer framing where physical layer signaling and pilot symbols are inserted to ease the estimation process at the receiver side. The output of this block is the PLFRAME. Finally, for shaping purposes, a classical Squared-Root Raised Cosine (SRRC) filter with variable roll-off factors $(0.2$, or 0.25 , or 0.35$)$ is considered.

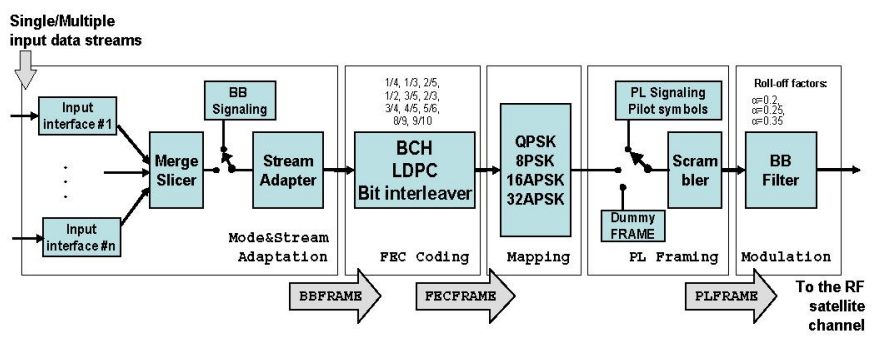

Figure 1 - DVB-S2 physical layer transmitter block diagram.

\section{B. Propagation Channel}

In this work, a time selective channel is considered. This assumption is in line with the generally accepted notion that in present satellite environments the direct component is generally strong, and multi-path power at resolvable delay is relatively very low and can be neglected with no accuracy loss. In order to stress the digital receiver with the aim to highlight the benefits of the HS-MRC scheme, a Rayleigh distributed satellite propagation channel is proposed. Moreover, an average terminal speed of $30 \mathrm{~km} / \mathrm{h}$ is assumed. With this low speed value, it is evident that the channel correlation time is sufficiently high to weaken the FEC correction capability if any diversity technique is taken into account

\section{Signal Model}

The base band received signal at any time instant can be modeled in vectorial form as:

$$
\mathbf{r}=\mathbf{h} s+\mathbf{n}
$$

where the time index have been dropped for the ease of notation, $\mathbf{h}$ is the $\mathrm{Nxl}$ vector associated to the satellite propagation channel, $N$ is the number of receive antennas, $s$ denotes the transmitted symbol with average transmitted power $P$ drawn out of the constellation selected by the adaptive modulation mechanism [1], and $\mathbf{n}$ stands for an additive Gaussian noise $N x I$ vector of complex, random variables with zero mean and variance $\sigma^{2}$. Prior to symbol detection, the contribution of the different receive antennas are combined by means of a beamforming vector, $\mathbf{w}$, as follows:

$$
y=\mathbf{w}^{T} \mathbf{r}
$$

It is well-known that the received signal-to-noise ratio (SNR) can be maximized by performing the Maximal Ratio Combining (MRC), i.e., $\mathbf{w}=\mathbf{h}^{*}$. Then, it can be easily proved that the instantaneous SNR can be written as:

$$
S N R_{M R C}=\frac{\|\mathbf{h}\|^{2} P}{\sigma^{2}}
$$

As the number of receive antennas is increased, so it is the received SNR. However, the involved complexity, size and cost may make the MRC approach prohibitive for practical implementation, especially in mobile terminals. For that reason, we adopt a low-complexity mechanism based on the HS-MRC mechanism [4]. Further details of the proposed mechanism are presented in the next Section.

\section{HYBRID SELECTION-MRC}

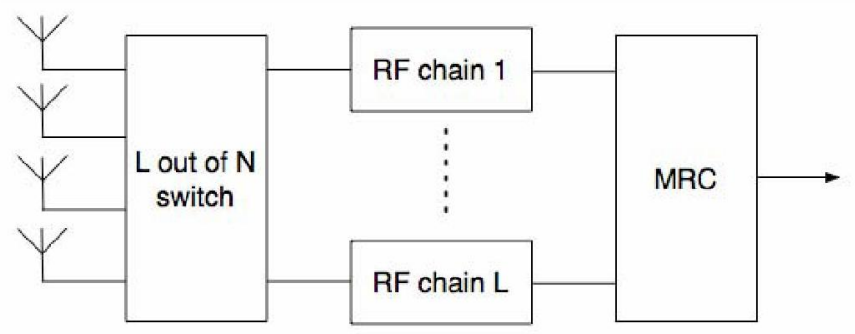

Figure 2 - Block diagram of the Hybrid Selection/MRC scheme

In a MRC reception scheme, better performance can be obtained by increasing the number of receive antennas. In doing so, however, the number of complete RF chains must also be increased and so it is the complexity and cost of the system. This negative effect can be drastically reduced by introducing an antenna selection mechanism. Instead of using all the receive antennas, only the best sub-set of them are selected for reception. In Figure 2, we show a reception scheme based on this reception procedure known as Hybrid Selection/MRC. The system is equipped with $N$ receive antennas, whereas a lower number of RF chains has been considered $(L \leq N)$. In accordance with the selection criterion, the best sub-set of receive antennas is selected. Once the subset of active antennas is selected, their contributions are combined by means of a MRC procedure.

As commented above, considerably savings in terms of complexity and cost of the system can be obtained with the Hybrid Selection/MRC strategy. This is because antenna elements and digital signal processing are considerably cheaper than introducing complete RF chains. As for system performance, it was shown that degradation observed in a wireless environment is slight in comparison with the saving in terms of hardware cost [4]. In this paper, we are devoted to quantify this lost in a satellite scenario when the system is focused on improving the link reliability of the system. In this case, the antenna selection mechanism consists in choosing the sub-set of $L$ receive antennas maximizing the received SNR. By taking into consideration that $L=2$, the received SNR of the HS-MRC approach can be written as:

$$
S N R_{H S-M R C}=\frac{P}{\sigma^{2}} \max _{1 \leq i, j \neq i \leq N}\left\{\left.h_{i}\right|^{2}+\left|h_{j}\right|^{2}\right\}
$$

Notice that the maximization is obtained by selecting the two receive antennas with the highest gains. As for the total number of available antennas, we will compare performance behavior for the cases with $N=2$ and 4 antennas. It is worth 
noting that these antennas should be placed by taking into consideration the spatial correlation distance to obtain the maximum benefits from this technique. Basically, the proposed mechanism selects the best paths for reception and, by doing so, the diversity order of the full antenna system can be extracted provided that antennas used in the selection procedure are uncorrelated [4]. Assuming directive antennas at $\mathrm{Ka}$ band $(20 \mathrm{GHz})$, the correlation distance is in the order of $0.15 \mathrm{~m}$. This value is reasonable for laptops, but maybe not for handheld devices. In this work we consider the first case and we leave the latter one for further research.

\section{Digital Receiver ARchitecture}

Several sub-systems are present in order to coherently demodulate and combine the received signal(s). Most of all estimation blocks in the DVB-S2 digital receiver could be dataaided (DA), exploiting the presence of a known preamble (PLHEADER) and several pilot fields allocated in the PLFRAME. In particular, in this article we discuss the Signalto-Noise ratio estimator (SNORE) [5], which is the circuit in charge of the selection of the best $L$ received paths.

We propose to perform the SNR estimation over the PLHEADER ( $N_{p}=90$ known symbols: 26 from SOF and 64 from re-encoding the MODCOD field) [1]. It shall be remembered that at 27.5 Mbaud, the PLHEADER lasts about $3.27 \mu \mathrm{s}$, that is sufficiently smaller than the channel coherence time, in order to keep constant the fading coefficient affecting the PLHEADER. In other words, the received PLHEADER samples can be expressed as follows:

$$
r_{k, j}=h_{j} s_{k}+n_{k, j}
$$

where $k$ is time index and $j$ indicates the receive antenna among $N$. In addition, $h_{j}$ is the fading amplitude affecting the PLHEADER of the $j$-th antenna, $s_{k}$ is the common transmitted preamble, and finally $n_{k, j}$ is the complex additive Gaussian noise with with zero mean and variance $\sigma^{2}$. According to that, the formulas for SNR estimation are [5]:

$$
\begin{aligned}
& \hat{P}_{S, j}=\left[\frac{1}{N_{p}} \sum_{k=1}^{N_{p}} \operatorname{Re}\left\{r_{k, j} s_{k}^{*}\right\}\right]^{2}=\left|h_{j}\right|^{2} P \\
& \hat{P}_{R, j}=\frac{1}{N_{p}} \sum_{k=1}^{N_{p}}\left|r_{k, j}\right|^{2}=\left|h_{j}\right|^{2} P-\sigma_{j}^{2} \\
& \hat{P}_{N, j}=\hat{P}_{R_{s}}-\hat{P}_{S_{, j}}=\sigma_{j}^{2} \\
& S N R_{j}=\frac{\hat{P}_{S, j}}{\hat{P}_{N, j}}=\frac{\left|h_{j}\right|^{2} P}{\sigma_{j}^{2}}
\end{aligned}
$$

Assuming the same noise variance for all receive antennas, the best $L$ antenna are chosen according to the estimate of the channel amplitude, Eq. (6), and the resulting SNR after HSMRC combining is reported in Eq. (4).
For the sake of completeness, the Eq. (10) computes the channel a-Priori information with combining $\mathrm{L}$ received sequences to correctly feed the DVB-S2 LDPC decoder:

$$
\log \left(\frac{\operatorname{Pr}\left\{b_{i}=0 \mid r_{k}\right\}}{\operatorname{Pr}\left\{b_{i}=1 \mid r_{k}\right\}}\right)=\log \left(\frac{\sum_{s_{i} \in S_{0}} \exp \left(-\sum_{j=0}^{L} \frac{\left|r_{k, j}-\hat{h}_{j} s_{i}\right|^{2}}{\sigma_{j}^{2}}\right)}{\sum_{s_{i} \in S_{1}} \exp \left(-\sum_{j=0}^{L} \frac{\left|r_{k, j}-\hat{h}_{j} s_{i}\right|^{2}}{\sigma_{j}^{2}}\right)}\right)
$$

where $S_{0}$ or $S_{1}$ is the set of symbols which have ' 0 ' or ' 1 ' in the $i$-th position, respectively.

\section{Simulation Results}

In this section, the HS-MRC scheme has been evaluated in the complete DVB-S2 transmit-receive chain. For this purpose, three different pairs of coding rate and modulation format have been selected: 1/4-QPSK, 1/2-QPSK, and 2/3-8PSK

The DVB-S2 detection performance with different values of $N$ and $L$ is depicted in Figure 3. Starting with the case $N=L=1$, no diversity technique is applied and it is evident that the channel is really challenging. To obtain $\mathrm{PER}=10^{-2}$, the SNR should be at least $20 \mathrm{~dB}$ for the 1/4-QPSK, and greater for the other configurations. Then, the second set of curves refers to the case $\mathrm{N}=\mathrm{L}=2$, the classical MRC scheme with two receive antennas. As expected, the gain is very large, in the order of 10 $\mathrm{dB}$ at $\mathrm{PER}=10^{-2}$, which is greater than the usual gain of $3 \mathrm{~dB}$ in AWGN channel when two paths are combined together. This result just confirms the well-known approach to introduce some diversity techniques to break the very long coherence time of the fading channel. Last but not least, the HS-MRC scheme with $\mathrm{L}=2$ over $\mathrm{N}=4$ is reported. Notably, the physical layer performance has experienced another significant and relevant improvement of about $7 \mathrm{~dB}$ at $\mathrm{PER}=10^{-3}$. This is an important result which shows that, although the propagation channel is not good (Rayleigh and slow terminal speed), it is feasible to achieve satisfactory results below $\mathrm{SNR}=10 \mathrm{~dB}$ also for medium/high efficiency scheme like 2/3-8PSK.

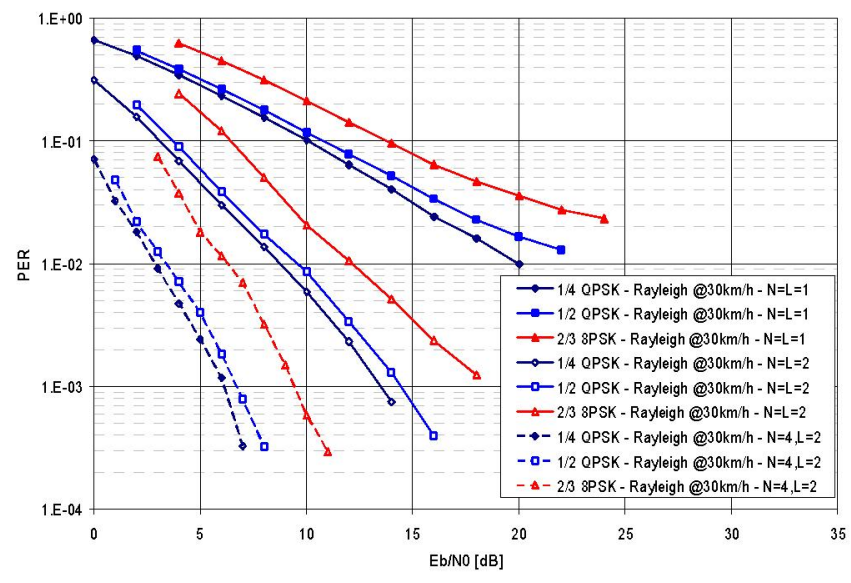

Figure 3 - Physical layer performance with several combinations of the parameters $L$ and $N$. 
For the sake of completeness, in Figure 4 the full complexity scheme, $\mathrm{N}=\mathrm{L}=4$, is compared with the HS-MRC scheme $\mathrm{N}=4-\mathrm{L}=2$. As observed in the figure, the diversity order of both schemes is the same as the curves present the same slope [4]. In other words, the antenna selection mechanism of the HS-MRC strategy is able of exploiting the diversity gain of the system with a lower level of complexity. Some performance is lost due to the fact that only two antennas are coherently combined but, however, this loss is minimal. More specifically, the figure shows that the distance in term of detection performance between the HS-MRC scheme $L=2-$ $N=4$ and the full complexity case $N=L=4$ is less than $1 \mathrm{~dB}$ at PER $=10^{-3}$.

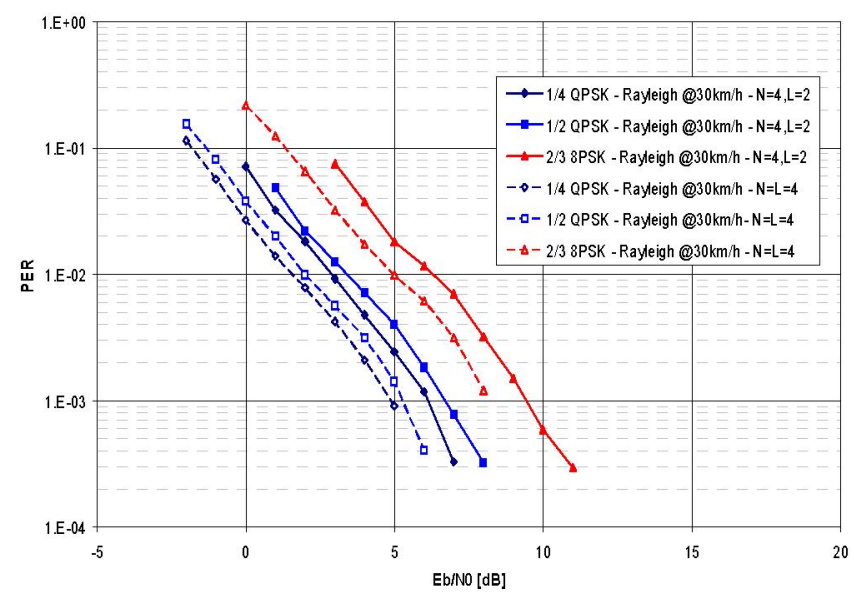

Figure 4 - Comparison between $\mathrm{L}=2-\mathrm{N}=4$ and $\mathrm{L}=\mathrm{N}=4$.

\section{CONCLUSIONS}

In this paper, we have proposed a Hybrid SelectionMaximal Ratio Combining approach to alleviate the impairments introduced by the LMS channel. This technique has been compared with several combinations of the parameter $L$ and $N$, in a Rayleigh fading channel with slow terminal speed, $30 \mathrm{~km} / \mathrm{h}$. The final statement is that the HS-MRC scheme $(L=2, N=4)$ is able to gain more than $6 \mathrm{~dB}$ with respect to the classical MRC approach with $\mathrm{N}=2$ receive antennas. Moreover, the performance loss with respect to the case $\mathrm{L}=\mathrm{N}=4$ is less than $1 \mathrm{~dB}$ at the price of a reduced receiver complexity.

Future investigation of this promising diversity technique is to verify and quantify possible benefits in spatial correlated fading channel.

\section{ACKNOWLEDGMENT}

This work was supported in part by the European Commission through SatNEx Network of Excellence IST507052 and by the Spanish Ministry of Science and Education MEC through project ESP2005-03403.

\section{REFERENCES}

[1] ETSI EN 302 307, "Digital video broadcasting (DVB); Second generation framing structure, channel coding and modulation systems for broadcasting, interactive services, news gathering and other broad-band satellite applications", 2006.

[2] S. Cioni, M.Berdondini, G.E. Corazza and A. Vanelli-Coralli, "Antenna Diversity for DVB-S2 Mobile Services in Railway Environments", Proc. of ASMS 2006 Conference, Herrsching (Germany), 29-31 May 2006.

[3] M. Alvárez Díaz, S. Scalise, D. Sciascia, R. Mura, P. Conforto and H. Ernst, "DVB-S Air Interface over Railroad Satellite Channel: Performance and Extensions", in Proc. of the $6^{\text {th }}$ Baiona Workshop on Signal Processing in Communications, Spain, Sep. 2003.

[4] M.Z. Win, and J.H. Winters, "Analysis of hybrid selection'maximal-ratiocombining in Rayleigh fading", IEEE Trans. on Communications, vol. 49, pp. 1926-1934, Nov. 2001.

[5] S. Cioni, G.E. Corazza, and M. Bousquet, "An Analytical Characterization of Maximum Likelihood Signal-to-Noise Ratio Estimation", Proc. of $2^{\text {nd }}$ International Symposium on Wireless Communications Systems (ISWCS), Siena (Italy), 5-9 Sep. 2005, vol. 1, pp. 827-830. 